\title{
A Área da Superfície Adesiva no Ensaio de Microtração Afeta a Resistência de União entre uma Cerâmica Feldspática e um Cimento Resinoso?
}

\author{
DDS, MSc, PhD, Professor Adjunto, Universidade Federal \\ de Santa Maria, Departamento de Odontologia \\ Restauradora, Santa Maria-RS, Brasil \\ (lfvalandro(a)hotmail.com) Endereço: Luiz Felipe \\ Luiz Felipe Valandro Valandro. Endereço: Universidade Federal de Santa \\ Maria, Faculdade de Odontologia, Departamento da \\ Odontologia Restauradora, Disciplina de Prótese, Rua \\ Marechal Floriano 1184, 97.015-372 Santa Maria-RS, \\ Brasil, Telefone: Telefone: 55-55-3220-9275 \\ DMD, PhD, Professora e Pesquisadora Associada, \\ Mutlu Özcan University Medical Center Groningen, University of \\ Groningen, Department of Dentistry and Dental Hygiene, \\ Groningen, The Netherlands \\ DDS, Mestranda em Prótese, Universidade Estadual \\ Regina Amaral Paulista, Faculdade de Odontologia de São Jose dos \\ Campos, São José dos Campos-SP, Brasil \\ DDS, MSc, doutoranda em Prótese, Universidade \\ Sheila Pestana Passos Fstadual Paulista, Faculdade de Odontologia de São Jose \\ dos Campos, São José dos Campos-SP, Brasil \\ DDS, PhD, Professor Adjunto, Universidade Estadual \\ Marco Antonio Bottino Paulista, Departamento de Materiais Dentários e Prótese, \\ São José dos Campos-SP, Brasil \\ Tipo de Análise do Manuscrito Triple Blind Peer Review \\ Recebido em Aprovado em
}

\section{RESUMO}

Objetivo: Avaliar o efeito da área da superfície de união na resistência adesiva entre cimento resinoso e cerâmica feldspática. Metodologia: Superfícies de 12 blocos cerâmicos $(6 \times 6 \times 4 \mathrm{~mm} 3)$ foram condicionadas com ácido fluorídrico $10 \%$ e silanizadas. Os blocos condicionados foram posicionados dentro de um molde de silicone e o cimento resinoso dual foi aplicado na superfície tratada. Os espécimes foram seccionados para obtenção de corpos-de-prova com diferentes áreas na superfície de união: Gr1 - 0,7mm x 0,7mm $\left(0,5 \mathrm{~mm}^{2}\right)$, Gr2 $-1 \mathrm{~mm} \times 1 \mathrm{~mm}\left(1 \mathrm{~mm}^{2}\right)$, Gr3 $-1,4 \mathrm{~mm} \times 1,4 \mathrm{~mm}\left(2 \mathrm{~mm}^{2}\right)$. Os corpos-de-prova foram submetidos ao teste de microtração em uma máquina de ensaio universal (velocidade: $1 \mathrm{~mm} / \mathrm{min}$ ). Os dados foram analisados estatisticamente (ANOVA e teste de Tukey; teste de correlação de Pearson). Resultados: Os valores de resistência foram influenciados significativamente pelas dimensões da área adesiva $(\mathrm{p}<, 0001)$. A maior média de resistência de união (MPa) foi a do $\operatorname{Gr} 1(26,3 a)$, seguida pelo Gr2 $(20,8 \mathrm{~b})$ e Gr3 $(16,1 c)$. O teste de correlação de Pearson revelou que há uma relação inversa entre a área da superfície de união e os resultados do teste de microtração $(r=-0,5234 ; p<0,0001)$. Conclusão: Quanto maior a área da superfície de união entre cerâmica e cimento resinoso, menor a resistência adesiva.

Palavras Chave: Resistência à tração, Cerâmica, Cimento 


\title{
L'adhésif de la surface d'essai sur microtension affecte la résistance
} de l'Union entre une céramique feldspathique et le ciment en résine?

\begin{abstract}
Purpose: To evaluate the effect of the bonded surface area on the bond strength of a resin cement to a glass ceramic. Methods: Surfaces of 12 glass ceramic blocks $(6 \times 6 \times 4 \mathrm{~mm} 3)$ were conditioned with 10\% hydrofluoric acid and silanizated. The conditioned blocks were placed inside a silicone mold and the dual-cure resin cement was applied on the treated surface. Specimens were stored sectioned for obtaining samples with different bonded surface areas: Gr1 - 0.7mm x 0.7mm $\left(0.5 \mathrm{~mm}^{2}\right)$, Gr2 - 1mm x 1mm $\left(1 \mathrm{~mm}^{2}\right)$, Gr3 - 1.4mm x $1.4 \mathrm{~mm}\left(2 \mathrm{~mm}^{2}\right)$. The non-trimmed beam samples were submitted to the microtensile bond strength test in a universal testing machine (cross-head speed: $1 \mathrm{~mm} / \mathrm{min}$ ). Data were statistically analyzed (ANOVA and Tukey's test; Pearson Correlation test). Results: The microtensile bond strength results were significantly affected by the bonded surface area ( $\mathrm{p}<.0001)$. The highest bond strength (MPa) was of the Gr1 $(26.3 \pm 8.2 \mathrm{a})$, followed by Gr2 $(20.8 \pm 6.5 \mathrm{~b})$ and Gr3 $(16.1 \pm 5.7 \mathrm{c})$. The Pearson correlation test revealed that there is an inverse correlation between bonded surface area and microtensile bond strength results $(r=-0.5234 ; p<0.0001)$. Conclusion: The lowest bonded surface areas provided the highest values of bond strength.
\end{abstract}

Keywords: Tensile bond strength, Ceramic, Cement.

\section{El adhesivo de superficie en la prueba microtracción afecta a la fuerza de la unión entre una cerámica feldespática y cemento de resina?}

\section{RESUMEN}

Objetivo: Evaluar el efecto de la superficie de matrimonio en la fuerza de adhesión entre el cemento de resina y de cerámica de feldespato. Metodología: 12 superficies de los bloques de cerámica $\left(6 \times 6 \times 4 \mathrm{~mm}_{3}\right)$ fueron acondicionadas con un $10 \%$ de ácido fluorhídrico y silanizada. Los bloques acondicionado se colocaron en un molde de silicona y el cemento de resina de doble se aplicó a la superficie tratada. Los especímenes fueron seccionados para obtener los órganos de probetas con diferentes áreas de la superficie de la unión: Gr1 - 0,7 mm x 0,7 mm (0,5 mm $\left.{ }^{2}\right)$, Gr2 - $\left.1 \mathrm{~mm} \times 1 \mathrm{~mm} \mathrm{(1} \mathrm{mm}{ }^{2}\right)$, Gr3 - 1,4 mm x 1,4 mm (2 $\mathrm{mm}^{2}$ ). Los cuerpos de la pieza de ensayo se sometió a pruebas microtracción en una máquina de prueba universal (velocidad: $1 \mathrm{~mm} / \mathrm{min}$ ). Los datos fueron analizados estadísticamente (ANOVA y prueba de Tukey, la prueba de correlación de Pearson). Resultados: Los valores de resistencia fueron significativamente influenciado por el tamaño de la zona del adhesivo ( $\mathrm{p}<0,0001$ ). La fuerza más largo enlace (MPa) fue el Gr1 $(26,3$ a), seguido por Gr2 $(20,8$ b) y Gr3 $(16,1 \mathrm{C})$. La prueba de correlación de Pearson reveló una relación inversa entre la superficie del matrimonio y los resultados de la prueba microtracción $(r=-0.5234, p<0,0001)$. Conclusión: Cuanto mayor es la superficie de unión entre la cerámica y el cemento de resina, menor será la resistencia de la unión.

Palabras clave: Resistencia a la tracción, Cerámica, Cemento

\section{L'adhésif de la surface d'essai sur microtension affecte la résistance de l'Union entre une céramique feldspathique et le ciment en résine?}

\section{RÉSUMMÉ}

Objectif: évaluer l'effet de la surface du mariage dans la force de liaison entre le ciment en résine et en céramique feldspathique. Méthodologie: 12 céramiques surfaces de blocs $(6 \times 6 \times 4 \mathrm{~mm} 3)$ ont été conditionnés avec de l'acide fluorhydrique à $10 \%$ et silanisée. Les blocs climatisées ont été placés dans un moule en silicone et le ciment en résine à double a été appliquée à la surface traitée. Les spécimens ont été sectionnés pour obtenir des 
organes éprouvette avec différents domaines sur la surface de l'union: Gr1 - 0,7 mm x 0,7 $\mathrm{mm}\left(0,5 \mathrm{~mm}^{2}\right)$, Gr2 - 1mm x 1mm (1mm $\left.{ }^{2}\right)$, Gr3 - 1,4 x mm 1,4 mm (2mm $\left.{ }^{2}\right)$. Les organes de l'éprouvette a été soumis à des tests microtension dans une machine d'essai universelle (vitesse: $1 \mathrm{~mm} / \mathrm{min}$ ). Les données ont été analysées statistiquement (ANOVA et test de Tukey, test de corrélation de Pearson). Résultats: Les valeurs de résistance ont été fortement influencés par la taille de la zone adhésive $(\mathrm{p}<.0001)$. La force la plus longue liaison (MPa) est Gr1 l'(26,3 a), suivi par Gr2 (20,8 b) et Gr3 (16,1 C). Le test de corrélation de Pearson a révélé une relation inverse entre la surface du mariage et les résultats de l'essai microtension $(r=-0,5234, p<0,0001)$. Conclusion: La plus grande est la surface de l'union entre la céramique et le ciment en résine, plus le la force de liaison.

Mots-clés: résistance à la traction, céramique, ciment

\section{Introdução}

Os testes de resistência de união têm sido utilizados para avaliar a eficácia da adesão do adesivo ao substrato (1). Muitos testes de resistência adesiva podem ser utilizados para diferentes substratos, tais como dentina-esmalte, resina composta, cerâmica, metal. Por exemplo, para avaliar a união da resina à superfície cerâmica, é possível realizar o teste de cisalhamento (2-4), tração convencional (4-6), ou microtração (corpos-de-prova em forma de ampulheta, alteres ou non-trimmed) $(1,7,8)$. A resistência à fadiga também pode ser usada para avaliar a união (9).

Quando uma investigação científica propõe testar a união entre um adesivo e um aderente, um dos aspectos essenciais é o método do teste empregado para mensurar a resistência na interface. Testes de resistência adesiva in vitro, como tração e cisalhamento, são baseados na aplicação da carga para gerar tensão no espécime até que ocorra a fratura. Portanto, para o teste promover valores de resistência de união adequados entre um adesivo e um substrato, é fundamental que a tensão seja maior na interface adesiva (1014). Muitos estudos que utilizaram análise de distribuição de tensão têm relatado que alguns ensaios de resistência de união não distribuem o esforço apropriadamente na zona adesiva (11,13-16). Testes de cisalhamento têm sido criticados devido à distribuição não homogênea da tensão na interface adesiva, induzindo uma interpretação equivocada dos resultados, visto que a falha se inicia em um dos substratos e não na zona adesiva $(\mathbf{1 1 , 1 3 -}$ 16). Embora, os testes de tração convencional também apresentem limitações similares, tais como dificuldade de alinhamento do espécime e a tendência de distribuição heterogênea da tensão $(\mathbf{1 1}, \mathbf{1 5})$, este tipo de teste pode ser empregado, pois provê informações da resistência adesiva global $(6,17,18)$. Por outro lado, o ensaio de microtração permite melhor alinhamento dos espécimes e uma distribuição mais homogênea da força, e, em adição, provê melhor comparação ou avaliação da resistência de união $(19,20)$.

O teste de microtração tem sido considerado um ensaio adequado para avaliar a resistência de união na interface entre cerâmica-cimento e uma variação da área da superfície dos espécimes para este teste pode modificar os resultados. Primeiramente, utilizando esmalte humano, Sano et al. (20) obtiveram uma relação inversa entre a área da superfície e os valores de resistência (maior superfície - menor resistência). Outros estudos, usando dente humano, notaram que a distribuição de tensão na superfície adesiva se modifica em função de variáveis como o tamanho e a forma da área adesiva do espécime $(21,22)$. Contudo, para o nosso conhecimento, não há nenhum estudo que investigou a influência da variação do tamanho da área da superfície adesiva na resistência entre cerâmica-resina de espécime non-trimmed. O objetivo deste estudo foi avaliar o efeito da área da interface adesiva na resistência de união de um cimento resinoso a uma cerâmica feldspática. A hipótese experimental é que a redução da área adesiva permite um aumento da resistência. 


\section{Material \& Métodos}

\section{Blocos cerâmicos}

Um molde de acetato foi usinado na dimensão de $8 \times 8 \times 6 \mathrm{~mm}$. Doze blocos cerâmicos (Vita VM7 Dentin 5M2, Vita Zahnfabrik, Bad Sackingen, Alemanha) foram preparados de acordo com as instruções do fabricante. A massa cerâmica foi inserida no molde e os blocos foram sinterizados em um forno (Vacumat, Vita Zahnfabrik) usando o programa específico indicado pelo fabricante. Em seguida, os blocos apresentaram aproximadamente $6,4 \times 6,4 \times 4,8 \mathrm{~mm} 3$, em virtude da contração de sinterização da cerâmica que é de aproximadamente $20 \%$. A superfície de cimentação $(6,4 \times 6,4 \mathrm{~mm})$ foi planificada e polida em uma máquina usando lixas d'água (3M, St. Paul, EUA) de granulações na seqüência $(600,800$ e 1200) sob refrigeração. O molde de cada bloco cerâmico foi obtido com silicone por reação de adição (Elite HD, Zhermack, Badia Polesine, Itália) sendo o bloco aprofundado dentro do silicone o suficiente para criar uma distância de $3 \mathrm{~mm}$ entre a porção superior do molde e a superfície do bloco. Posteriormente, o cimento foi injetado dentro deste espaço.

\section{Tratamento da superfície}

Antes do condicionamento e silanização, todos os blocos foram limpos em ultrassom (Vitasonic, Vita Zanhfabrik) por 5 min usando água destilada. As superfícies cerâmicas foram condicionadas com ácido fluorídrico gel a 10\% (Dentsply, Petrópolis, Brasil) por 1 min, lavados com jato ar-água e secos. Os blocos foram limpos com água destilada em ultrassom por $4 \mathrm{~min}$. Em seguida, foi aplicada uma camada de silano (Porcelain Primer, Bisco, Schaumburg, EUA).

\section{Aplicação do cimento}

Cada bloco cerâmico tratado foi posicionado em seu molde de silicone. O cimento resinoso (Variolink II, Ivoclar-Vivadent, Schaan, Liechtenstein) foi manipulado de acordo com as recomendações do fabricante e injetado dentro do molde sobre a superfície tratada do bloco cerâmico, usando uma seringa do tipo centrix (DFL, Rio de Janeiro, Brasil).

A superfície exposta do cimento foi polimerizada dentro do molde (XL 3000, 3MESPE, St Paul, EUA; $500 \mathrm{~mW} / \mathrm{cm} 2$ ) por $40 \mathrm{~s}$. Após $10 \mathrm{~min}$, o conjunto bloco cerâmico cimento resinoso foi removido de dentro do molde e o cimento foi novamente submetido à polimerização das cinco faces (superior e laterais) por 40 s cada lado (23). Os blocos foram lavados com água e armazenados em água destilada a $37^{\circ} \mathrm{C}$ por $24 \mathrm{~h}$ antes da preparação dos espécimes.

\section{Preparação dos espécimes para o teste de microtração}

Os blocos de cerâmica-cimento foram seccionados usando um disco de diamante (n. 34570; Microdont, São Paulo, Brasil) em uma velocidade baixa, sob refrigeração $(8,24)$. Inicialmente, os blocos cimentados foram fixados com adesivo cianoacrilato gel (Super Bonder Gel, Loctite Ltd, São Paulo, Brasil) na base metálica que foi fixada na máquina de corte. Os blocos foram posicionados perpendicularmente em relação ao disco diamantado da máquina. A primeira secção de aproximadamente $1 \mathrm{~mm}$ foi descartada em caso da possível presença de excesso ou ausência de cimento na interface que poderia alterar os resultados. Posteriormente, três secções foram obtidas com aproximadamente $0.7 \mathrm{~mm}$, $1 \mathrm{~mm}$ e $1.4 \mathrm{~mm}$ de espessura. Cada secção foi rotacionada em $90^{\circ}$ e novamente fixada na base metálica. A primeira secção foi descartada $(\mathbf{1} \pm 0, \mathbf{1 m m})$ por causa das razões já descritas anteriormente. Outras secções de $0,7 \mathrm{~mm}, 1 \mathrm{~mm}$, e $1,4 \mathrm{~mm}$ foram obtidas respectivamente (Figura 1). 


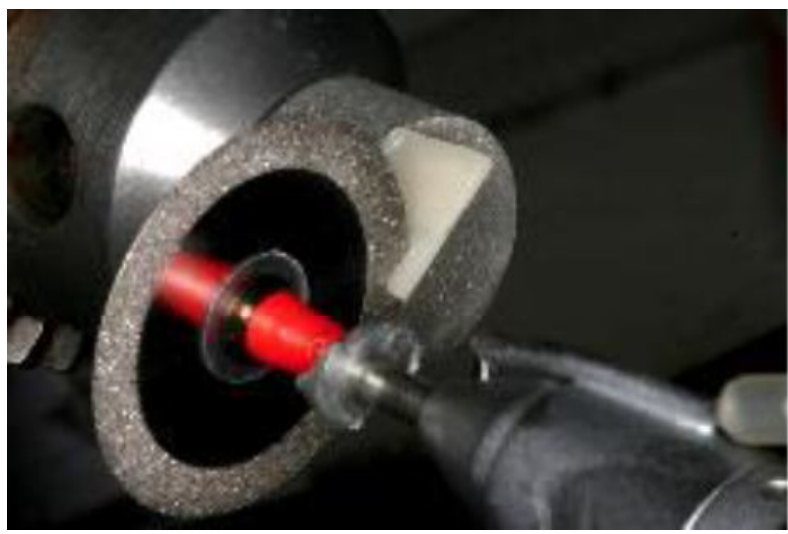

Figura 1. Obtenção dos espécimes non-trimmed.

Portanto, três grupos de espécimes non-trimmed foram obtidos, considerando o tamanho da área adesiva: Gr1: área adesiva de aproximadamente $0,5 \mathrm{~mm}^{2}(0,7 \mathrm{~mm} \times$ 0,7mm); Gr2: área adesiva de aproximadamente $1 \mathrm{~mm}^{2}$ (1mm x 1mm); Gr3: área adesiva de aproximadamente $2 \mathrm{~mm}^{2}(1,4 \mathrm{~mm} \times 1,4 \mathrm{~mm})$

\section{Teste de microtração}

Cada espécime foi fixado com cianoacrilato gel nas hastes de um dispositivo para realização do ensaio, deixando a zona adesiva livre. Os espécimes foram posicionados paralelamente ao longo eixo do dispositivo, a fim de reduzir possíveis forças de torção. $O$ dispositivo foi fixado em uma máquina de ensaio universal (EMIC DL-10oo, EMIC, São José dos Pinhais, Brasil), numa posição paralela à força de tração aplicada, e o teste foi realizado a uma velocidade de $1 \mathrm{~mm}$. min-1. A resistência adesiva " $\mathrm{R}$ " (MPa) foi calculada de acordo com a fórmula $R=F / A$, onde " $F$ " é a força solicitada para ruptura do espécime $(N)$ e "A" é a área da interface do espécime $(\mathrm{mm} 2)$, mensurada com o auxílio de um paquímetro digital antes do ensaio (Figura 2).

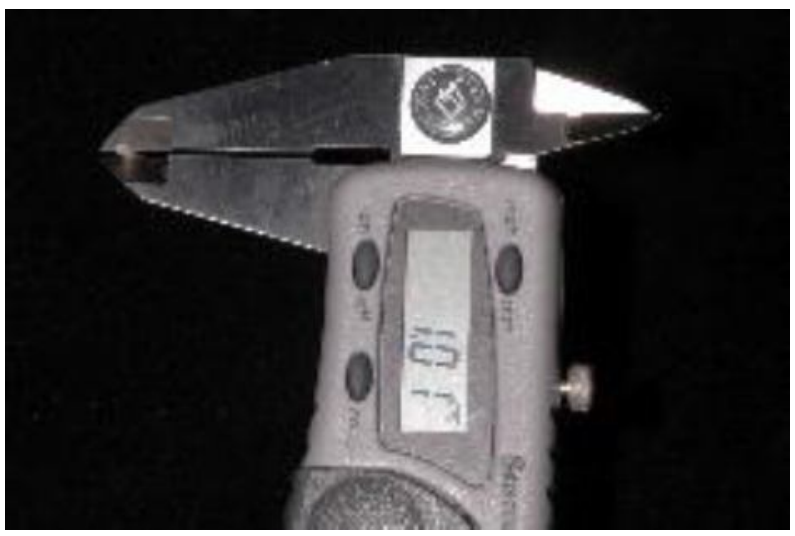

Figura 2. Mensuração da espessura dos espécimes com paquímetro digital. 


\section{Análise estatística}

A análise estatística foi realizada usando o software Statistix 8.0 for Windows (Analytical Software Inc, Tallahassee, FL, EUA). Os dados da resistência adesiva (MPa) foram submetidos à análise de variância a um critério e teste de Tukey $(\square=0,05)$. A análise da correlação de Pearson foi usada para verificar a correlação entre o tamanho da área e os resultados obtidos com o ensaio de microtração. Os valores da área da superfície também foram analisados estatisticamente, considerando três diferentes áreas (One-way ANOVA e Tukey, $\square=0,05$ ).

\section{Análise do modo de falha}

Todos os espécimes submetidos ao teste de microtração foram analisados sob microscopia óptica (Stemi 2000-C, Carl Zeiss, Jena, Alemanha) a uma magnificação de $50 x$ para verificar se a fratura ocorreu na zona adesiva. Baseado no estudo de Della Bona et al. (25), a zona adesiva no presente estudo consistiu em: cimento resinoso e a região da interface entre o cimento resinoso e a cerâmica feldspática.

A superfície fraturada de alguns espécimes representativos foi selecionada para análise através da microscopia eletrônica de varredura (MEV) (1000x - 2000x) para observação dos tipos de falha entre: a cerâmica e o cimento (ADES); falha coesiva no cimento (COES-cim); falha coesiva na cerâmica (COES-cer); falha coesiva no cimento combinado com falha na interface cerâmica-cimento (MISTA).

\section{Resultados}

ANOVA a um critério (Tabela 1 ) revelou que a área adesiva afetou significativamente a resistência no ensaio de microtração $(\mathrm{P}<0,001)$. O teste de Tukey por múltiplas comparações $(\alpha=0,05)$ mostrou que Gr1 $(26,3 \pm 8,1 \mathrm{a})$ obteve a maior resistência adesiva (MPa) quando comparado com os grupos Gr2 $(20,8 \pm 6,5 \mathrm{~b})$ e $\mathrm{Gr} 3(16,1 \pm 5,7 \mathrm{c})$ (Figura 3 ). Portanto, a hipótese experimental foi aceita.

Tabela 1

Resultados da ANOVA 1 critério

\begin{tabular}{l|c|c|c|c|c}
\hline Source & df & SS & MS & F & P \\
\hline Between & 2 & 2622.81 & 1311.41 & 27.5 & $<0.0001$ \\
\hline Within & 147 & 6997.93 & 47.6 & & \\
\hline Total & 150 & 10752 & & & \\
\hline
\end{tabular}




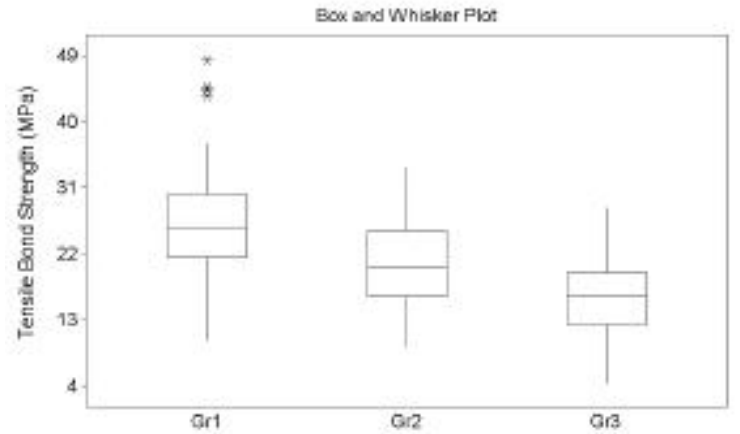

Figura 3 Médias e desvio padrão dos dados de resistência adesiva (MPa).

A análise de correlação de Pearson (Figura 4) revelou que existe uma relação inversa $(\mathrm{r} 2=-0,5237 ; \mathrm{P}<0,0001)$ entre a área e os valores de resistência. Para tanto, quanto maior a área, menor os valores de resistência de união.

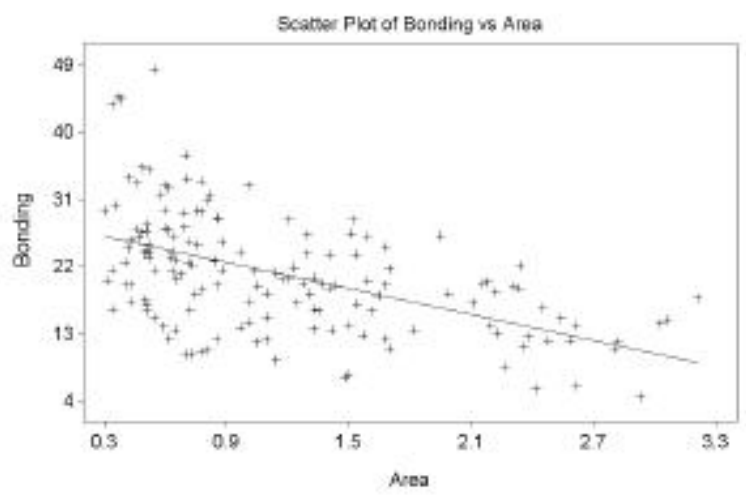

Figura 4 Scatter Plots for the correlação entre a área da superfície de união em mm2 (eixo x) e valores da resistência a tração em MPa (eixo y).

O ANOVA a um critério confirmou que os valores da área (mm2) dos três grupos foram diferentes significativamente $(\mathrm{P}<.0001)(\mathrm{Gr} 1[0,5 \pm 0,1 \mathrm{a}]>\mathrm{Gr} 2[1 \pm 0,2 \mathrm{~b}]>\mathrm{Gr} 3$ $[2 \pm 0,5 \mathrm{c}])$.

O tipo de falha sob microscopia óptica mostrou que todas as fraturas dos espécimes ocorreram na zona adesiva. Através do MEV, independente do tamanho da secção transversal da área de superfície, $\mathbf{1 0 0 \%}$ das falhas foram mistas - combinadas com falhas coesivas do cimento com falhas adesivas da interface cimento-cerâmica (Figura 5). 


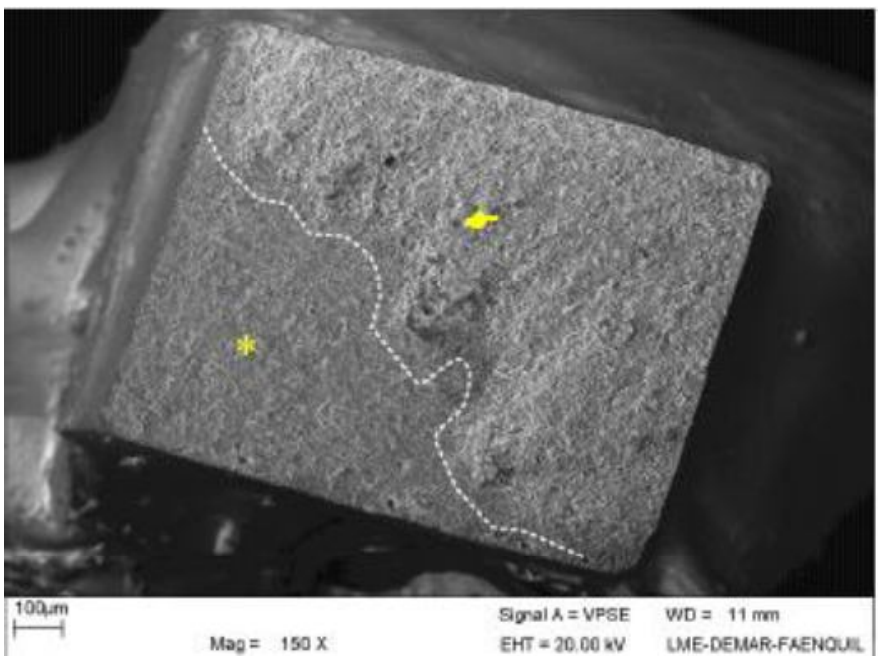

Figura $5 \mathrm{a}-\mathrm{b}$ Micrografias representativas das falhas mistas. Os $\square$ pontos fora da fratura da superficie de cimento, indicando falha coesiva do cimento resinoso ${ }^{*}$ indica a superfície coesiva da cerâmica, mostrando as falhas na interface cerâmica-cimento.

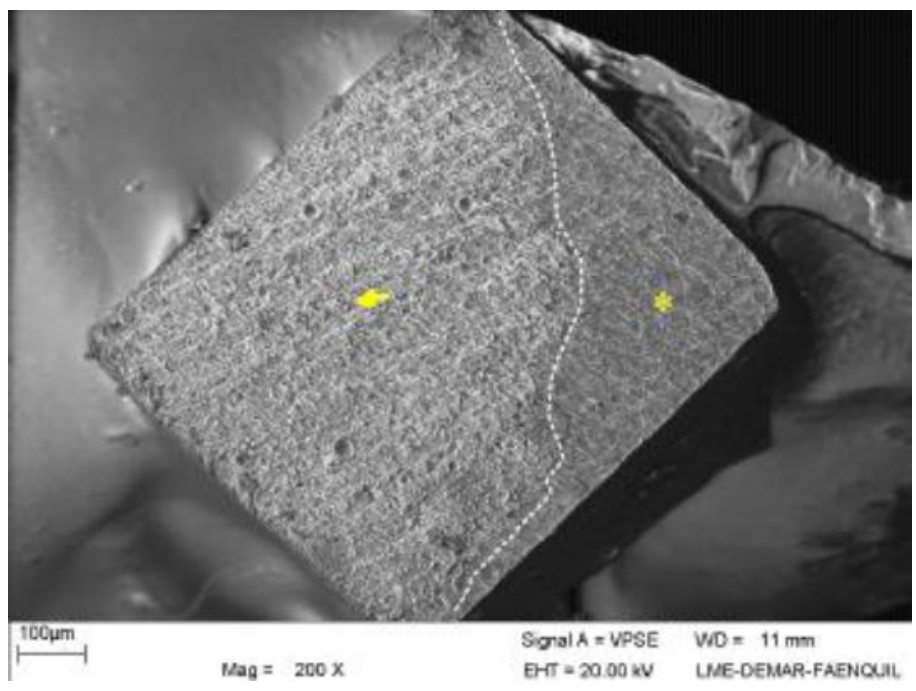

\section{Discussão}

O método utilizado para avaliação da união a um substrato está diretamente relacionado ao desempenho desta adesão; o que significa que os resultados da resistência adesiva dependem do tipo de teste empregado. De acordo com alguns estudos que avaliaram a distribuição da força ao longo da interface adesiva, o teste de cisalhamento promove tensões não uniformes, gerando maior tensão sobre o substrato e introduzindo erros na avaliação da resistência de união entre adesivo e aderente (11-16). Isto é explicado pelo fato que tensões são concentradas em áreas restritas fora da interface, produzindo maior potencial de falha coesiva na base do material à partir de um ponto crítico de concentração do esforço $(\mathbf{1 1}, 16)$. O teste de microtração tem mostrado distribuição mais homogênea de tensões, permitindo melhor comparação ou avaliação da resistência adesiva $(19,20)$. Entretanto, variações de forma ou tamanho da área de superfície dos espécimes para o teste de microtração podem influenciar os resultados da resistência $(20-22,26)$.

Este estudo observou que os resultados da resistência adesiva pelo teste de microtração em uma cerâmica feldspática dependem da área da secção transversal do 
espécime non-trimmed. O teste de correlação de Pearson indicou uma relação inversa entre estes dois fatores; a menor área da superfície foi associada a uma maior resistência a tração, enquanto a maior área mostrou menor resistência a tração. Esses resultados concordam com o de outros estudos $(20-22,26)$, e revelam que a padronização do tamanho da área da superfície do espécime non-trimmed para o teste de microtração é obrigatória, especialmente para que possam ser comparados os resultados entre diferentes estudos.

Sano et al. (20), usando dentina humana, avaliaram a relação entre a área da superfície na adesão e nos resultados de tração para dois sistemas adesivos e um ionômero, e observaram que todas as estratégias utilizadas reduziram os resultados de resistência adesiva quando a área foi aumentada. Usando dentina humana, Phrukkanon et al. (22) observaram resultados semelhantes aos de Sano et al. (20), demonstrando a relação inversa entre área e os valores de resistência a tração e ao cisalhamento. Phrukkanon et al. (21), utilizaram a análise de elemento finito para avaliar a distribuição de tensão em espécimes com aproximadamente 1,1, 1,5 e 3,1 $\mathrm{mm} 2$ de área na superfície de união. Espécimes com interface cilíndrica e retangular foram comparados. Considerando ambas as formas, o espécime com menor área de superfície foi capaz de distribuir o estresse melhor que os espécimes maiores. Os espécimes cilíndricos distribuíram o estresse na superfície melhor que os espécimes retangulares. Nos espécimes cilíndricos o estresse foi homogeneamente distribuído ao redor da periferia da superfície de união, enquanto que nos espécime retangulares o estresse se concentrou nos cantos e na área central das faces. Considerando a área da secção transversal, os autores observaram que o máximo de estresse ocorreu nos espécimes com 1,1 mm2 sendo de 42,13 MPa, enquanto que no de 3,1 $\mathrm{mm} 2$ foi de 57,54 MPa. Considerando o mecanismo de fratura (27), o mais importante destes resultados é que a área com maior concentração de estresse será a área onde a trinca inicia e propaga até ocorrer fratura catastrófica do espécime. Portanto, o espécime que expressa menor tensão máxima tem maior chance de sobreviver durante o teste de tração

Uma explicação possível para os resultados do presente estudo é que a menor área transversal melhora a distribuição de esforço durante o teste (22). Outra razão para este fenômeno pode ser o efeito da presença de defeitos na interface ou no substrato. Considerando a teoria de Griffith (28), a resistência à tração de um material uniforme diminui quando o tamanho do espécime é aumentado. Este resultado pode ocorrer em função da distribuição dos defeitos nos espécimes maiores, que provavelmente têm mais defeitos que espécimes menores. Como a falha de materiais frágeis se inicia em algum defeito, este aumento pode acrescer o risco de ocorrer uma falha.

Por outro lado, áreas pequenas da secção transversal exibem menores valores de carga em Newton (N) e os resultados em MPa são maiores. Enquanto que maiores áreas exibem maiores valores de carga em Newton (N), e resultados em MPa menores. A partir destes aspectos, a chance de falhas pré-teste é mais alta com espécimes menores que com os maiores. Neste estudo, notou-se que quando foi realizado o corte do bloco para obtenção dos espécimes, 25 espécimes de $0.5 \mathrm{~mm} 2$ foram perdidos, enquanto que apenas 5 espécimes com $1 \mathrm{~mm} 2$ fraturaram e nenhum espécime com $2 \mathrm{~mm} 2$ fraturou. Estes resultados apontam que pequenas áreas de secção transversal podem ser desvantajosas. Após análise dos resultados, pode-se recomendar que espécimes non-trimmed com área de aproximadamente $\mathbf{1} \mathrm{mm} 2$ podem ser usados para avaliação da resistência adesiva no teste de microtração entre cimento resinoso e cerâmicas feldspáticas.

Outros estudos de resistência de união realizaram a análise do tipo de falha do espécime $(6,17,19,20,29,30)$. Se o propósito da investigação for a união entre o adesivo e um aderente, um dos aspectos fundamentais é que a fratura ocorra na interface entre adesivo-aderente e não coesivamente. Testes de cisalhamento têm sido criticados devido à distribuição não-homogênea do esforço na interface adesiva. Portanto, a falha freqüientemente se inicia em um dos substratos e não na zona adesiva, induzindo muitas falhas coesivas e uma subestimação ou uma interpretação equivocada dos resultados (11- 
16). No presente estudo, todas as fraturas foram semelhantes, ocorrendo na zona adesiva, independente do grupo. Não ocorreu nenhuma falha coesiva fora da zona adesiva.

\section{Conclusão}

As menores áreas apresentaram maiores valores de resistência adesiva à uma cerâmica feldspática. Pode ser observada a existência de uma tendência significante: quando a superfície da área adesiva aumenta, a resistência de união à cerâmica diminui.

\section{Referências}

1. Pashley DH, Carvalho RM, Sano H, Nakajima M, Yoshiyama M, Shono Y, et al. The microtensile bond test: a review. J Adhes Dent 1999;1:299-309.

2. Awliya W, Odén A, Yaman P, Dennison JB, Razzoog ME. Shear bond strength of a resin cement to densely sintered high-purity alumina with various surface conditions. Acta Odontol Scand 1998;56:9-13.

3. Blixt M, Adamczak E, Lindén L, Odén A, Arvidson K. Bonding to densely sintered alumina surfaces: effect of sandblasting and silica coating on shear bond strength of luting cements. Int $J$ Prosthodont 2000;13:221-26.

4. Özcan M, Alkumru H, Gemalmaz D. The effect of surface treatment on the shear bond strength of luting cement to a glass-infiltrated alumina ceramic. Int J Prosthodont 2001;14:335-39.

5. Wegner SM, Kern M. Long-term resin bond strength to zirconia ceramic. J Adhes Dent 2000;2:139-47.

6. Della Bona A, Anusavice KJ, Hood JAA. Effect of ceramic surface treatment on tensile bond strength to a resin cement. Int $J$ Prosthodont 2002;15:248-53.

7. Shono Y, Terashita M, Shimada J, Kozono Y, Carvalho RM, Russel CM, et al. Durability of resindentin bond. J Adhes Dent 1999;1:211-18.

8. Valandro LF, Özcan M, Bottino MC, Scotti R, Bottino MA, Della Bona A. Bond strength of a resin cement to high-alumina and zirconia-reinforced ceramics: the effect of surface conditioning. $J$ Adhes Dent 2006;8:175-81.

9. Baldissara P, Valandro LF, Monaco C, Ferrari M, Bottino MA, Scotti R. Fatigue resistance of the bond of a glass-infiltrated alumina ceramic to the human dentin. J Adhes Dent 2006;8:97-104.

10. Anusavice KJ, DeHoff PH, Fairhurst CW. Comparative evaluation of ceramic-metal bond tests using finite element stress analysis. J Dent Res 1980, 59(3):608-613.

11. Van Noort R, Noroozi S, Howard IC, Cardew G. A critique of bond strength measurements. J Dent 1989, 12(2):61-67.

12. Van Noort R, Cardew G, Howard IC, Noroozi S. The effect of local interfacial geometry on the measurement of the tensile bond strength to dentin. J Dent Res 1991, 7O(5):889-893.

13. Della Bona A, Van Noort R. Shear vs. tensile bond strength of resin composite bonded to ceramic. J Dent Res 1995, 74(9):1591-1596.

14. DeHoff PH, Anusavice KJ, Wang Z. Three-dimensional finite element analysis of the shear bond test. Dent Mater 1995, 11(2):126-131.

15. Kitasako Y, Burrow MF, Nikaido T, Harada N, Inokoshi S, Yamada T, et al. Shear and tensile bond testing for resin cement evaluation. Dent Mater 1995, 11(5):298-304.

16. Versluis A, Tantbirojn D, Douglas WH. Why do shear bond tests pull out dentin? J Dent Res 1997, 76(6):1298-1307.

17. Chen KK, Shono Y, Ogawa T, Kozono Y, Terashita M. Fracture aspects of resin-dentin bonding in non-trimming microtensile test. Dent Mater J 2001, 2O(4):315-324.

18. Hooshmand T, Van Noort R, Keshvad A. Bond durability of resin-bonded and silane treated ceramic surface. Dent Mater 2002, 18(2):179-188.

19. Della Bona A, Anusavice KJ, Shen C. Microtensile strength of composite bonded to hot-pressed ceramics. J Adhes Dent 2000, 2(4):305-313. 
20. Sano H, Shono T, Sonoda H, Takatsu T, Ciucchi B, Carvalho R, et al. Relationship between surface area for adhesion and tensile bond strength - Evaluation of a micro-tensile bond test. Dent Mater 1994, 10(4):236-240.

21. Phrukkanon S, Burrow MF, Tyas MJ. Effect of cross-sectional surface area on bond strengths between resin and dentin. Dent Mater 1998a, 14(2):120-128.

22. Phrukkanon S, Burrow MF, Tyas MJ. The influence of cross-sectional shape and surface area on the microtensile bond test. Dent Mater 1998b, 14(3):212-221.

23. Foxton RM, Pereira PNR, Nakajima M, Tagami J, Miura H. Durability of the dual-cure resin cement/ceramic bond with different curing strategies. J Adhes Dent 2002, 4(1):49-59.

24. Amaral R, Bottino MA, Valandro LF, Özcan M. Microtensile bond strength of a resin cement to glass infiltrated zirconia-reinforced ceramic: the effect of surface conditioning. Dent Mater 2006, 22(3):283-290.

25. Della Bona A, Anusavice KJ, Mecholsky Jr JJ. Failure analysis of resin composite bonded to ceramic. Dent Mater 2003, 19:693-699.

26. Shono Y, Terashita M, Pashley EL, Brewer PD, Pashley DH. Effects of cross-sectional area on resin-enamel tensile bond strength. Dent Mater 1997, 13(5):290-296.

27. Gdoutos EE. Fractures mechanics - An introduction. Boston, MA: Kluwer Academic Publishers, 1-14, 1993.

28. Griffith AA. The phenomena of rupture and flow in solids. Phil Trans R Soc London, Ser. A 221: 168-198, 1920.

29. Mecholsky Jr J. Fracture mechanics principles. Dent Mater 1995, 11(2):111-112.

30. Mecholsky Jr J. Fractography: determining the sites of fracture initiation. Dent Mater 1995, 11(2):113-116. 